\title{
LFG Monitoring Deployment Planning Based on Distributed Compressing Sensing Method
}

\author{
Qian Zhang and Chuan Huang* \\ State Key Laboratory of Coal Mine Disaster Dynamics and Control, Chongqing University, College of Resources and \\ Environmental Science, Chongqing University, Chongqing 400044, China \\ ${ }^{*}$ Corresponding author
}

\begin{abstract}
To minimize the hazard landfill gas (LFG), a monitoring scheme depending on wireless sensors network (WSN) was designed, and information reconstruction was developed to obtain optimal accuracy in the terminal. In this model, the gasguide area was divided into different clusters. Then, we present an improved joint sparsity model based on distributed compressing sensing method was designed. And compressing sensing system within a sparse measurement matrix and a new wavelet tree model-based adaptive iterative hard thresholding recovery algorithm were also presented. Simulation results show that the scheme can efficiently accomplish monitoring planning.
\end{abstract}

Keywords—distributed compressing sensing; monitoring; WSN

\section{INTRODUCTION}

Along with city development and population growth in China, the quantity of municipal waste raise by leaps. Landfill has become the main means to deal with municipal waste and it will be exist as the final disposal method in the long run [1]. Then landfill gas (LFG) is the main product from relevant reactions. It consists of four primary components, one of which is methane [2]. Methane from landfills is a big problem which has potential hazards associated because of the flammability [3]. Therefore, the special issues of the landfill must be supervision such as the air pressure of gas-guide tube. In fact, these parameters was gathered by manual measurement in one-time operation periodically, but the workload is so heavy as well as the behaviour is dangerous. In this way, we came up with monitoring scheme based on wireless sensors network instead of superfluous manual tasks.

\section{LFG MONITORING DEPLOYMENT PLANNING}

In this work, we propose a new approach aiming to achieve an efficient procedure to monitor the status of LFG. Following the experience gained in several monitoring contexts, we found that deployments of inexpensive wireless sensor network with full automatic is appropriate for a large-scale area to deal with vast variety of data [4]. Nowadays, sensor set on the top of tubes for receiving the monitoring data. The rationale of the monitoring plan proposed in this work is outlined in Figure I . In general, the first stage is data acquisition, all the monitoring data is collected by masses of wireless sensors. Information will be captured and transmitted via wireless communication to the terminal for store and analysis. While after the data transfer procedure, data is uploaded and stored in dedicated database server, and is ready for the following processing steps.

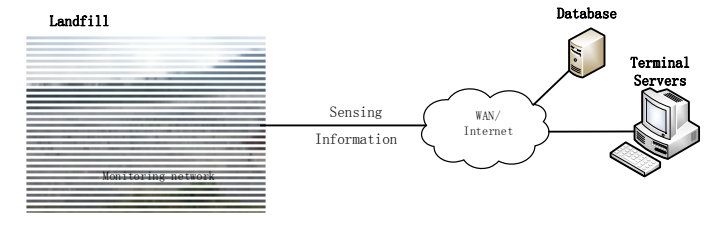

FIGURE I. THE SCHEME FOR MONITORING IN A LANDFILL

In practice, the gas-tubes were placed into the equilateral triangle shape for better coverage. Two tubes in a fixed interval of 25-30 metres with practice experience in the completed landfill [5]. So the data captured have correlation coefficients between each other. We put forward a modified model based on distributed compressing sensing method on account of the correlation information among gas-tubes. A joint sparsity model which make the algorithm more applicable to the largescale landfills in order to optimize the transmission. Nodes connect each other, however, the communication boundary energy and computer capacity of a single sensor are limited. When the WSN is constituted by thousands of tiny node in a large monitoring area, the sensing information of identical kind is correlation. So we present a model with picking up a cluster node to reduce transinformation so as to economize time and energy. Entire process of the proposed approach is provided in detail below. Figure II is a sample image between cluster and nodes.

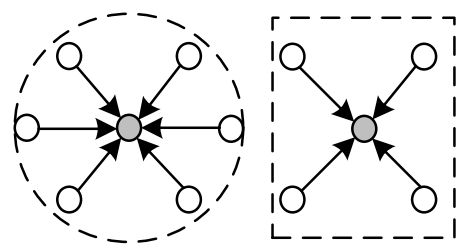

FIGURE II. COMMUNICATION BETWEEN CLUSTER AND NODES IN GROUPS

The herein more detailed key elements of the system of the procedure step-by-step is behind, presented methods referred to a monitoring network based on wireless sensor networks (WSNs). 


\section{TRANSMISSION ALGORITHM BASED ON DISTRIBUTED COMPRESSING SENSING}

\section{A. Overview of the Transmission Algorithm}

Compressing sensing apply to the wireless sensors network due to its lower complexity. However, the monitoring system expects real-time and lower fault tolerance. Where, the first step of the data compression is preprocessed in a cluster. Meanwhile, the proposed reconstruction algorithm which has faster convergence speed and similar error bound when compared with some other compressive sensing methods is improved. The MAIHT scheme has the following advantages: (1) combining IHT and wavelet tree model algorithms to obtain higher reconstruction accuracy and (2) using an adaptive parameter $\mu$ to speed up the convergence.

Each node in one group transmits data to the cluster at regular intervals. Cluster node save the original signal as a matrix, as in (1),

$$
\mathrm{X}=\left[\begin{array}{llll}
x_{1} & x_{2} & \cdots & x_{\mathrm{M}}
\end{array}\right]=\left[\begin{array}{cccc}
x_{11} & x_{12} & \cdots & x_{1 \mathrm{M}} \\
x_{21} & x_{22} & \cdots & x_{2 \mathrm{M}} \\
\cdots & \cdots & \cdots & \cdots \\
x_{\mathrm{N} 1} & x_{\mathrm{N} 2} & \cdots & x_{\mathrm{NM}}
\end{array}\right]
$$

Aiming to sparsified the original $\mathrm{X}$ with help of the wavelet transformation that can be expressed by $x_{i}=\Psi \alpha_{i}, i=1,2, \cdots, \mathrm{M}$. $\alpha_{i}, i=1,2, \cdots, \mathrm{M}$ is the sparsity coefficients. Depending on the processor, the joint representation is given

$$
\alpha_{i}=\omega_{c}+\omega_{i}, i=1,2, \ldots, \mathrm{M}
$$

where $\omega_{c}$ is the joint component of all coefficients and $\omega_{i}$ is the characteristic component. We minimize the sparsity of the joint representation by solving optimization problem

$$
\min \left(\left\|\omega_{c}\right\|_{0}+\left\|\omega_{1}\right\|_{0}+\ldots+\left\|\omega_{\mathrm{M}}\right\|_{0}\right) \text { s.t. } \alpha_{i}=\omega_{c}+\omega_{i}, i=1,2, \ldots, \mathrm{M} \text {. }
$$

Hence we

$$
\mathrm{B}=\left[\begin{array}{llll}
\omega_{c} & \omega_{1} & \ldots & \omega_{\mathrm{M}}
\end{array}\right]
$$

According to the sparsity of joint component and characteristic component, the corresponding number of the measurement obtained and matrixes $\Phi_{s}, \Phi_{1}, \Phi_{2}, \ldots, \Phi_{\mathrm{M}}$ generated. The M-dimension measurement matrix $\Phi$ were Random Gaussian matrix. Measure process in the cluster is expressed by

$$
\mathrm{Y}=\left[\begin{array}{cccc}
\Phi_{s} & 0 & \cdots & 0 \\
0 & \Phi_{1} & \cdots & 0 \\
\cdots & \cdots & \cdots & \cdots \\
0 & \cdots & 0 & \Phi_{M}
\end{array}\right] \mathrm{B}
$$

which the measurement matrix

$$
\mathrm{Y}=\left[\begin{array}{lllll}
y_{s} & y_{1} & y_{2} & \cdots & y_{\mathrm{M}}
\end{array}\right]
$$

At last, cluster translates the measurement matrix Y.

Model-based Adaptive Hard Threshold Iteration (MAIHT) method is used in terminal to reconstruct each signal of the original X. The proposed algorithm is summarized as follows:

- Initialize:

$\mathrm{X}_{1}=0$, residual $\mathbf{r}=\mathbf{y}$, iterations $n=0$;

Supports $\Lambda=\varnothing, J=\varnothing$;

- While halting criterion false do

$n=n+1$;

$\mathrm{X}_{n+1}=\mathrm{X}_{n}+\mu \Phi^{\mathrm{T}} \mathrm{r}, \mu=\frac{\left\|\mathrm{x}_{\mathrm{k}}-\mathrm{X}_{\mathrm{k}-1}\right\|_{2}^{2}}{\left\|\Phi\left(\mathrm{x}_{\mathrm{k}}-\mathrm{x}_{\mathrm{k}-1}\right)\right\|_{2}^{2}} ;$

$J \leftarrow \mathrm{M}\left(\mathrm{x}_{n+1}, 2 m\right)$, getting support according to signal model $\mathrm{M}, 2 \mathrm{~m}$ maximum indexes;

$T=\operatorname{supp}\left(\mathrm{x}_{n}\right) \cup J$, merging supports;

$\left.b\right|_{T}=\Phi_{T}^{\dagger} y$, signal estimating;

$\mathrm{x}_{n+1} \leftarrow \mathrm{M}(b, m)$,pruning signal estimate $b$ with $m$ maximum indexes;

$\mathbf{r}_{\text {new }}=\mathbf{y}-\boldsymbol{\Phi}_{n+1}$,updating measurement residual;

- $\quad$ End while

return $\mathrm{X}=\mathrm{X}_{n}$.

\section{B. Simulation of Algorithm}

To quantitatively evaluate the proposed method, we simulate experiment for the algorithm described before including comparing the proposed method with some mature algorithms and accuracy between joint sparse model and separate reconstruction. We quantify reconstruction performance of the algorithm in terms of root mean square error (RMSE). The RMSE denotes the root mean square error estimated between the original signal and the recovery result. For an original signal $\mathrm{X}$ of length $\mathrm{N}$, The RMSE is given by (6). 


$$
R M S E=\sqrt{\frac{1}{N} \sum_{i=1}^{N}\left(X_{i}-X_{i}^{\prime}\right)^{2}}
$$

where $X_{i}$ and $X_{i}^{\prime}$ represent the original signal and the recovery result, respectively. In the practical implementation, a large number of iterations were set. The RMSE index are temporarily stored. Final result is the mean value of them.

The analysis of performance comparison in terms of RMSE is shown. First of all, computational complexity is calculated by these parameters: according to the randomness in the process, convergence time is an average value of 500 times.

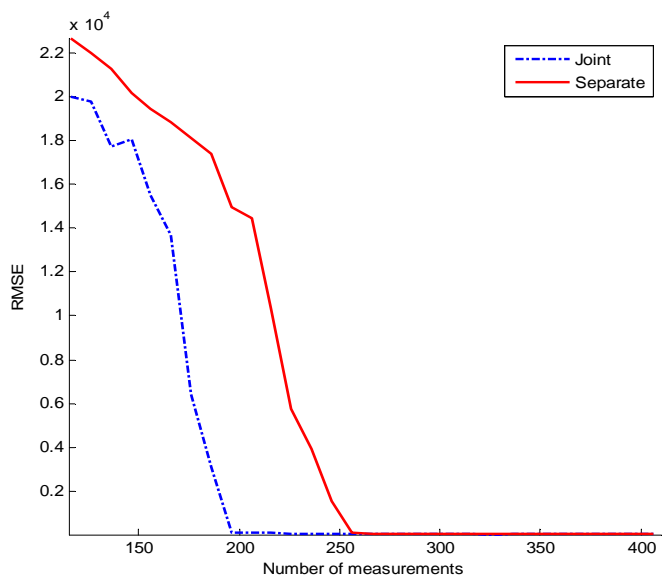

FIGURE III. COMPARISON BETWEEN RECONSTRUCTION OF JOINT SPARSITY MODEL AND SEPARATE RECONSTRUCTION

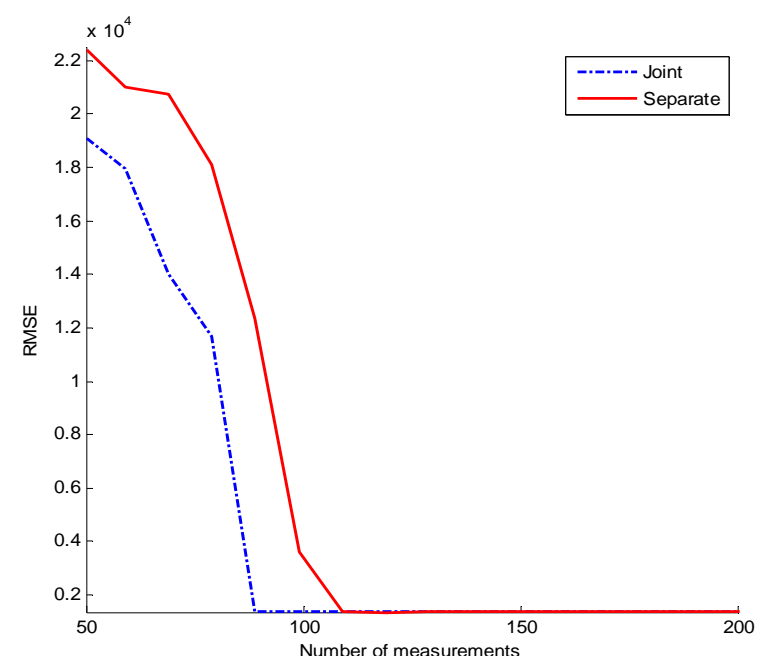

FIGURE IV. COMPARISON BETWEEN JOINT SPARSITY MODEL AND SEPARATE WITH LESS DATA

Experiments results between joint sparsity model and separate reconstruction poste in Figure III and Figure IV. We start simulation by the model with two nodes and one cluster node, and the length of the test data is 1600. Figure III shows calculation that compared with refactoring separately, our method can reconstruct one original signal in a higher accuracy with less measurements. Furthermore, in order to verify effectiveness of the model by four more nodes and one cluster, and the length of data reduced to 640 proportionally. It is obvious to see in Figure IV that the model with more nodes can also guarantee an accurate reconstruction and require less measurements.

Simulation result shows that the proposed algorithm have nice performance, and the RMSE are improved in the same precondition. With the less measurement numbers, our proposed joint sparsity model based on distributed compressing sensing method can reach the minimal RMSE first of all. In conclusion, the experiments mentioned above show that the proposed algorithm and model are consistently slightly better.

\section{CONCLUSIONS}

This paper presented a monitoring system for the landfills, solved engineering problems such like manual work. Firstly, monitor the whole scenario through assigning a variety of wireless sensors in gas-guide area. Moreover, CS-based compression was found to WSN due to its lower complexity and best overall energy efficiency. This paper proposed a joint sparsity model which is suitable for observing landfills on account of the correlation information among gas tubes. CSbased system within a sparse measurement matrix and a new model-based adaptive iterative hard thresholding recovery algorithm is expressed to improve the transmission effectively and precisely of mass data. As expected, it is superior to the others in robustness in simulation verification.

In our future work, we will manufacture the equipment and lay out networks in a large scale landfill to implement monitoring in reality.

\section{ACKNOWLEDGMENT}

This research work was supported by the 2014 National Technology Support Project of China (No.2014BAC29B01). The authors are grateful to Prof. G. Liu. We express our appreciation to the anonymous reviewers.

\section{REFERENCES}

[1] Arigala S G, Tsotsis T T, Yortsos Y C, et al. Gas Generation, Transport, and Extraction in Landfills[J]. Journal of Environmental Engineering, 1995, 121(1):33-44.

[2] Peter Kjeldsen, Morton A. Barlaz, Alix P. Rooker, et al. Present and Long-Term Composition of MSW Landfill Leachate: A Review[J]. Critical Reviews in Environmental Science \& Technology, 2002, 32(4):297-336

[3] Townsend T G, Wise W R, Jain P. One-Dimensional Gas Flow Model for Horizontal Gas Collection Systems at Municipal Solid Waste Landfills [J]. Journal of Environmental Engineering, 2005, 131(12): págs. 1716-1723.

[4] Stark T D, Choi H. Methane gas migration through geomembranes[C]// 2005:120-125.

[5] Vigneault H, Lefebvre R, Nastev M. Numerical Simulation of the Radius of Influence for Landfill Gas Wells[J]. Vadose Zone Journal, 2004, 3(3):909-916

[6] Richard G. Baraniuk, Volkan Cevher, Marco F. Duarte, Chinmay Hegde, Model-Based Compressive Sensing, IEEE Transactions on, 2010, vol.56, pp. 1982-2001. 
[7] Baron D, Duarte M F, Sarvotham S, et a.l Distributed compressed sensing of jointly sparse signals [C]. MProc 39th Asilo 2 mar Conf Signals, Systems and Computers. Pacific Grove, CA, 2005 : 1537-1541.

[8] Liu G, Zhang Q, et al. Model-Based Adaptive Iterative Hard Thresholding Compressive Sensing in Sensor Network for Volcanic Earthquake Detection [J]. International Journal of Distributed Sensor Networks, 2015, 2015(3). 